Sādhanā Vol. 29, Part 1, February 2004, pp. 83-92. @ Printed in India

\title{
Response of orthotropic micropolar elastic medium due to time harmonic source
}

\author{
RAJNEESH KUMAR and SUMAN CHOUDHARY \\ Mathematics Department, Kurukshetra University, Kurukshetra 136 119, India \\ e-mail: \{rajneesh_kuk, sumanmaths\}@ rediffmail.com
}

MS received 15 March 2002; revised 13 November 2002

\begin{abstract}
The present paper is concerned with the plane strain problem in homogeneous micropolar orthotropic elastic solids. The disturbance due to time harmonic concentrated source is investigated by employing eigen-value approach. The integral transforms have been inverted by using a numerical technique to obtain the component of displacement, force stress and couple stress in the physical domain. The results of these quantities are given and illustrated graphically.
\end{abstract}

Keywords. Micropolar; orthotropic; eigenvalue; time harmonic source.

\section{Introduction}

In many engineering phenomena, including the response of soils, geological materials and composites, the assumptions of an isotropic behaviour may not capture some significant features of the continuum response. The formulation and solution of anisotropic problems is far more difficult and cumbersome than its isotropic counterpart. In the last years the elastodynamic response of anisotropic continuum has received the attention of several researchers. In particular, transversely isotropic and orthotropic materials, which may not be distinguished from each other in plane strain and plane stress cases, have been more regularly studied.

The theory of micropolar elasticity introduced and developed by Eringen (1966) aroused much interest because of its possible utility in investigating the deformation properties of solids for which the classical theory is inadequate. The micropolar theory is believed to be particularly useful in investigating materials consisting of bar-like molecules which exhibit microrotation effects and which can support body and surface couples. Recently, Cheng \& He (1995,1997), Erbay (2000) and Kumar \& Deswal (2000, 2001) have studied different problems in micropolar isotropic medium.

A review of literature on micropolar orthotropic continua shows that Iesan (1973, 1974a, 1974b) analyzed the static problems of plane micropolar strain of a homogeneous and orthotropic elastic solid, torsion problem of homogeneous and orthotropic cylinders in the linear theory of micropolar elasticity and bending of orthotropic micropolar elastic beams by terminals couple. Nakamura et al (1984) derived finite element method for orthotropic micropolar elasticity.

Most of the problems studied so far, in micropolar elasticity, involve the use of potential functions. However, the use of the eigen-value approach has the advantage of finding the 
solutions of equations in the coupled form directly, in the matrix notations, where as the potential function approach requires decoupling of equations. Yet, the eigen-value approach has not been applied in micropolar orthotropic medium. Mahalanabis \& Manna (1989) applied eigen-value approach to linear micropolar elasticity by arranging basic equations of linear micropolar elasticity in the form of matrix differential equation. Mahalanabis \& Manna (1997) discussed the problem of linear micropolar thermoelasticity by using eigen-value approach. Recently Kumar et al (2001) applied the eigen-value approach to micropolar elastic medium due to impulsive force at origin.

\section{Problem formulation}

We consider a homogeneous and orthotropic micropolar medium of infinite extent with Cartesian co-ordinate system $(x, y, z)$. To analyse displacement and stresses at the interior of the medium due to time harmonic concentrated load, the continuum is divided into two halfspaces defined by:

(i) half-space I $|x|<\infty,-\infty<y \leq 0$

(ii) half-space II $|x|<\infty, 0 \leq y<\infty$.

A time harmonic source is assumed to be acting on the interface of two half-spaces $(y=0)$ in the medium. We apply the arbitrary load $F_{o}(x, t)=F_{o}(x) \mathrm{e}^{i \omega t}$.

If we restrict our analysis parallel to $x y$-plane with displacement vector $\mathbf{u}=\left(u_{1}, u_{2}, 0\right)$ and microrotation vector $\phi=\left(0,0, \phi_{3}\right)$, the basic equations in the dynamic theory of the plane strain of homogeneous and orthotropic micropolar solids in absence of body forces and body couples, given by Eringen (1968), can be recalled as:

$$
\begin{aligned}
t_{j i, j} & =\rho \frac{\partial^{2} u_{i}}{\partial t^{2}}, \\
m_{i 3, i}+\epsilon_{i j 3} t_{i j} & =\rho j \frac{\partial^{2} \phi_{3}}{\partial t^{2}} .
\end{aligned}
$$

The constitutive relations, given by Iesan (1973), can be written as:

$$
\begin{array}{rlrl}
t_{11}= & A_{11} \epsilon_{11}+A_{12} \epsilon_{22}, & t_{12} & =A_{77} \epsilon_{12}+A_{78} \epsilon_{21}, \\
t_{21}= & A_{78} \epsilon_{12}+A_{88} \epsilon_{21}, & t_{22} & =A_{12} \epsilon_{11}+A_{22} \epsilon_{22}, \\
m_{13}=B_{66} \phi_{3,1}, & m_{23} & =B_{44} \phi_{3,2},
\end{array}
$$

where

$$
\epsilon_{i j}=u_{j, i}+\epsilon_{j i 3} \phi_{3} .
$$

In these relations, we have used the following notations: $t_{i j}$ - components of the force stress tensor, $m_{i j}$ - component of the couple stress tensor, $\epsilon_{i j}$ - component of micropolar strain tensor, $u_{i}$ - components of displacement vector, $\phi_{3}$ - component of microrotation vector, $\epsilon_{i j k}$ - permutation symbol, $A_{11}, A_{12}, A_{22}, A_{77}, A_{78}, A_{88}, B_{44}, B_{66}$ - characteristic constants of the material, $\rho$ - the density and $j$ - the microinertia.

From (1)-(4), we obtain the field equations of the plane strain for orthotropic micropolar solids in the form:

$$
\left(A_{11} \frac{\partial^{2}}{\partial x^{2}}+A_{88} \frac{\partial^{2}}{\partial y^{2}}\right) u_{1}+\left(A_{12}+A_{78}\right) \frac{\partial^{2} u_{2}}{\partial x \partial y}-K_{1} \frac{\partial \phi_{3}}{\partial y}=\rho \frac{\partial^{2} u_{1}}{\partial t^{2}},
$$




$$
\begin{aligned}
&\left(A_{12}+A_{78}\right) \frac{\partial^{2} u_{1}}{\partial x \partial y}+\left(A_{77} \frac{\partial^{2}}{\partial x^{2}}+A_{22} \frac{\partial^{2}}{\partial y^{2}}\right) u_{2}-K_{2} \frac{\partial \phi_{3}}{\partial x}=\rho \frac{\partial^{2} u_{2}}{\partial t^{2}}, \\
&\left(B_{66} \frac{\partial^{2}}{\partial x^{2}}+B_{44} \frac{\partial^{2}}{\partial y^{2}}-\chi\right) \phi_{3}+K_{1} \frac{\partial u_{1}}{\partial y}+K_{2} \frac{\partial u_{2}}{\partial x}=\rho j \frac{\partial^{2} \phi_{3}}{\partial t^{2}},
\end{aligned}
$$

where

$$
K_{1}=A_{78}-A_{88}, \quad K_{2}=A_{77}-A_{78}, \quad \chi=K_{2}-K_{1} .
$$

Assuming time harmonic behaviour as

$$
\begin{aligned}
& u_{i}(x, y, t)=u_{i}(x, y) e^{i \omega t}, ; i=1,2, \\
& \phi_{3}(x, y, t)=\phi_{3}(x, y) e^{\imath \omega t} .
\end{aligned}
$$

Introducing the dimensionless quantities

$$
\begin{aligned}
x^{*}=\frac{x}{h}, \quad y^{*}=\frac{y}{h}, \quad u_{i}^{*} & =\frac{u_{i}}{h}, \\
\phi_{3}^{*}=\frac{A_{11}}{K_{1}} \phi_{3}, \quad t_{i j}^{*}=\frac{t_{i j}}{A_{11}}, \quad t^{*} & =\sqrt{\frac{A_{11}}{\rho h^{2}} t} \\
m_{i j}^{*}=\frac{h}{B_{66}} m_{i j}, \quad \omega^{* 2} & =\frac{\rho h^{2}}{A_{11}} \omega^{2},
\end{aligned}
$$

where $h$ is the parameter of dimension of length.

Using (7) and (8), the system of equations (5) reduce to (dropping the asterisks for convenience)

$$
\begin{gathered}
\left(A_{11} \frac{\partial^{2}}{\partial x^{2}}+A_{88} \frac{\partial^{2}}{\partial y^{2}}\right) u_{1}+\left(A_{12}+A_{78}\right) \frac{\partial^{2} u_{2}}{\partial x \partial y}-\frac{K_{1}^{2}}{A_{11}} \frac{\partial \phi_{3}}{\partial y}=-A_{11} \omega^{2} u_{1} \\
\left(A_{12}+A_{78}\right) \frac{\partial^{2} u_{1}}{\partial x \partial y}+\left(A_{77} \frac{\partial^{2}}{\partial x^{2}}+A_{22} \frac{\partial^{2}}{\partial y^{2}}\right) u_{2}-\frac{K_{1} K_{2}}{A_{11}} \frac{\partial \phi_{3}}{\partial x}=-A_{11} \omega^{2} u_{2} \\
\left(B_{66} \frac{\partial^{2}}{\partial x^{2}}+B_{44} \frac{\partial^{2}}{\partial y^{2}}-h^{2} \chi\right)\left(\frac{K_{1}}{A_{11} h^{2}}\right) \phi_{3}+K_{1} \frac{\partial u_{1}}{\partial y}+K_{2} \frac{\partial u_{2}}{\partial x}=-\frac{j K_{1}}{h^{2}} \omega^{2} \phi_{3} .
\end{gathered}
$$

Applying Fourier transform w.r.t ' $x$ ' defined by

$$
\left\{\tilde{u}_{i}(\xi, y, t), \tilde{\phi}_{3}(\xi, y, t)\right\}=\int_{-\infty}^{\infty}\left\{u_{i}(x, y, t), \phi_{3}(x, y, t)\right\} e^{\iota \xi x} d x, i=1,2,
$$

on (9)-(11), we obtain

$$
\begin{aligned}
& \tilde{u}_{1}^{\prime \prime}=Q_{11} \tilde{u}_{1}+Q_{15}{\tilde{u^{\prime}}}_{2}+Q_{16}{\tilde{\phi^{\prime}}}_{3}, \\
& \tilde{u}_{2}=Q_{22} \tilde{u}_{2}+Q_{23} \tilde{\phi}_{3}+Q_{24}{\tilde{u^{\prime}}}_{1}, \\
& {\tilde{\phi^{\prime \prime}}}_{3}=Q_{32} \tilde{u}_{2}+Q_{33} \tilde{\phi}_{3}+Q_{34}{\tilde{u^{\prime}}}_{1},
\end{aligned}
$$


where

$$
\begin{aligned}
& Q_{11}=\frac{A_{11}\left(\xi^{2}-\omega^{2}\right)}{A_{88}}, Q_{15}=\frac{\iota \xi\left(A_{12}+A_{78}\right)}{A_{88}}, Q_{16}=\frac{K_{1}^{2}}{A_{11} A_{88}}, \\
& Q_{22}=\frac{\left(\xi^{2} A_{77}-\omega^{2} A_{11}\right)}{A_{22}}, Q_{23}=-\frac{\iota \xi K_{1} K_{2}}{A_{11} A_{22}}, Q_{24}=\frac{\iota \xi\left(A_{12}+A_{78}\right)}{A_{22}}, \\
& Q_{32}=\frac{\iota \xi h^{2} K_{2} A_{11}}{B_{44} K_{1}}, Q_{33}=\frac{\left(\xi^{2} B_{66}+\chi^{2} h^{2}\right)-j \omega^{2} A_{11}}{B_{44}}, Q_{34}=-\frac{h^{2} A_{11}}{B_{44}} .
\end{aligned}
$$

The system of equations (13)-(15) can be written as

$$
\frac{\mathrm{d}}{\mathrm{d} y} W(\xi, y, \omega)=A(\xi, \omega) W(\xi, y, \omega),
$$

where

$$
\begin{array}{cc}
W=\left[\begin{array}{c}
U \\
U^{\prime}
\end{array}\right], \quad A=\left[\begin{array}{cc}
O & I \\
A_{2} & A_{1}
\end{array}\right], \quad U=\left[\begin{array}{c}
\tilde{u}_{1} \\
\tilde{u}_{2} \\
\tilde{\phi}_{3}
\end{array}\right], \quad O=\left[\begin{array}{lll}
0 & 0 & 0 \\
0 & 0 & 0 \\
0 & 0 & 0
\end{array}\right], \\
I=\left[\begin{array}{lll}
1 & 0 & 0 \\
0 & 1 & 0 \\
0 & 0 & 1
\end{array}\right], A_{1}=\left[\begin{array}{ccc}
0 & Q_{15} & Q_{16} \\
Q_{24} & 0 & 0 \\
Q_{34} & 0 & 0
\end{array}\right], A_{2}=\left[\begin{array}{ccc}
Q_{11} & 0 & 0 \\
0 & Q_{22} & Q_{23} \\
0 & Q_{32} & Q_{33}
\end{array}\right],
\end{array}
$$

To solve (17), we take

$$
W(\xi, y, \omega)=X(\xi, \omega) e^{q y}
$$

so that

$$
A(\xi, \omega) W(\xi, y, \omega)=q W(\xi, y, \omega)
$$

which leads to eigen-value problem. The characteristic equation corresponding to the matrix $A$ is given by

$$
\operatorname{det}[A-q I]=0,
$$

which on expansion provides us

$$
q^{6}-\lambda_{1} q^{4}+\lambda_{2} q^{2}-\lambda_{3}=0,
$$

where

$$
\begin{aligned}
\lambda_{1}= & Q_{15} Q_{24}+Q_{16} Q_{34}+Q_{11}+Q_{22}+Q_{33}, \\
\lambda_{2}= & Q_{15}\left(Q_{24} Q_{33}-Q_{23} Q_{34}\right)+Q_{16}\left(Q_{22} Q_{34}-Q_{24} Q_{32}\right) \\
& +Q_{11} Q_{22}+Q_{22} Q_{33}+Q_{11} Q_{33}-Q_{23} Q_{32}, \\
\lambda_{3}= & Q_{11}\left(Q_{22} Q_{33}-Q_{23} Q_{32}\right) .
\end{aligned}
$$

The roots of (22) are $\pm q_{i}, i=1,2,3$. 
The eigen values of the matrix $A$ are the roots of (22). We assume that real parts of $q_{i}$ are positive. The vector $\mathbf{X}(\xi)$ corresponding to the eigen values $q_{i}$ can be determined by solving the homogeneous equation

$$
[A-q I] X(\xi, \omega)=0 .
$$

The set of eigen vectors $\mathbf{X}_{\mathbf{i}}(\xi, \omega),(i=1,2,3,4,5,6)$ may be obtained as

$$
X_{i}(\xi, \omega)=\left[\begin{array}{l}
X_{i 1}(\xi, \omega) \\
X_{i 2}(\xi, \omega)
\end{array}\right]
$$

where

$$
\begin{aligned}
X_{i 1}(\xi, \omega) & =\left[\begin{array}{c}
a_{i} q_{i} \\
b_{i} \\
1
\end{array}\right], X_{i 2}(\xi, \omega)=\left[\begin{array}{c}
a_{i} q_{i}^{2} \\
b_{i} q_{i} \\
q_{i}
\end{array}\right], q=q_{i} ; i=1,2,3 \\
X_{j 1}(\xi, \omega) & =\left[\begin{array}{c}
-a_{i} q_{i} \\
b_{i} \\
1
\end{array}\right], X_{j 2}(\xi, \omega)=\left[\begin{array}{c}
a_{i} q_{i}^{2} \\
-b_{i} q_{i} \\
-q_{i}
\end{array}\right], \\
j & =i+3, q=-q_{i} ; i=1,2,3 \\
a_{i} & =\left(q_{i}^{2} Q_{15}+Q_{16} Q_{32}-Q_{15} Q_{33}\right) / \Delta_{i}, \\
b_{i} & =\left[q_{i}^{4}-q_{i}^{2}\left(Q_{16} Q_{34}+Q_{11}+Q_{33}\right)+Q_{11} Q_{33}\right] / \Delta_{i}, \\
\Delta_{i} & =q_{i}^{2}\left(Q_{15} Q_{34}+Q_{32}\right)-Q_{32} Q_{11} .
\end{aligned}
$$

The solution of (17) is given by

$$
W(\xi, y, \omega)=\sum_{i=1}^{3}\left[B_{i} X_{i}(\xi, \omega) \exp \left(q_{i} y\right)+B_{i+3} X_{i+3}(\xi, \omega) \exp \left(-q_{i} y\right)\right],
$$

where, $B_{i}(i=1,2,3,4,5,6)$ are arbitrary constants.

Equation (30) represents the solution of the general problem in the plane strain case of micropolar orthotropic elasticity by employing the eigen-value approach and therefore can be applied to a broad class of problem in the domain of Fourier transforms.

\section{Application}

We consider an infinite micropolar orthrotropic space in which a concentrated force of magnitude $F_{o}(x, t)=F_{o} \delta(x) \mathrm{e}^{t \omega t}$, acting in the direction of the $y$-axis at the origin of the Cartesian co-ordinate system. The boundary conditions at the interface of two half-spaces $(y=0)$ are given by

$$
\begin{aligned}
& u_{1}\left(x, 0^{+}, t\right)-u_{1}\left(x, 0^{-}, t\right)=0, \quad u_{2}\left(x, 0^{+}, t\right)-u_{2}\left(x, 0^{-}, t\right)=0, \\
& \phi_{3}\left(x, 0^{+}, t\right)-\phi_{3}\left(x, 0^{-}, t\right)=0, \quad m_{23}\left(x, 0^{+}, t\right)-m_{23}\left(x, 0^{-}, t\right)=0, \\
& t_{21}\left(x, 0^{+}, t\right)-t_{21}\left(x, 0^{-}, t\right)=0, \\
& t_{22}\left(x, 0^{+}, t\right)-t_{22}\left(x, 0^{-}, t\right)=-F_{o} \delta(x) e^{i \omega t} .
\end{aligned}
$$


Using (7) and (8) and then applying Fourier transforms from (12) on the system of equations (31), we get

$$
\begin{aligned}
& \tilde{u}_{1}\left(\xi, 0^{+}, \omega\right)-\tilde{u}_{1}\left(\xi, 0^{-}, \omega\right)=0, \quad \tilde{u}_{2}\left(\xi, 0^{+}, \omega\right)-\tilde{u}_{2}\left(\xi, 0^{-}, \omega\right)=0, \\
& \tilde{\phi}_{3}\left(\xi, 0^{+}, \omega\right)-\tilde{\phi}_{3}\left(\xi, 0^{-}, \omega\right)=0, \quad \tilde{m}_{23}\left(\xi, 0^{+}, \omega\right)-\tilde{m}_{23}\left(\xi, 0^{-}, \omega\right)=0, \\
& \tilde{t}_{21}\left(\xi, 0^{+}, \omega\right)-\tilde{t}_{21}\left(\xi, 0^{-}, \omega\right)=0, \\
& \tilde{t}_{22}\left(\xi, 0^{+}, \omega\right)-\tilde{t}_{22}\left(\xi, 0^{-}, \omega\right)=-F_{o} .
\end{aligned}
$$

The transformed displacement, microrotation and stresses are given for $y \geq 0$ as

$$
\begin{aligned}
\tilde{u}_{1}(\xi, y, t) & =-\left(a_{1} q_{1} B_{4} e^{-q_{1} y}+a_{2} q_{2} B_{5} e^{-q_{2} y}+a_{3} q_{3} B_{6} e^{-q_{3} y}\right) e^{\imath \omega t} \\
\tilde{u}_{2}(\xi, y, t) & =\left(b_{1} B_{4} e^{-q_{1} y}+b_{2} B_{5} e^{-q_{2} y}+b_{3} B_{6} e^{-q_{3} y}\right) e^{i \omega t} \\
\tilde{\phi}_{2}(\xi, y, t) & =\left(B_{4} e^{-q_{1} y}+B_{5} e^{-q_{2} y}+B_{6} e^{-q_{3} y}\right) e^{\imath \omega t} \\
\tilde{m}_{23}(\xi, y, t) & =-\left(B_{44} K_{1} / B_{66} A_{11}\right)\left(q_{1} B_{4} e^{-q_{1} y}+q_{2} B_{5} e^{-q_{2} y}+q_{3} B_{6} e^{-q_{3} y}\right) e^{\imath \omega t} \\
\tilde{t}_{21}(\xi, y, t) & =\left(M_{1} B_{4} e^{-q_{1} y}+M_{2} B_{5} e^{-q_{2} y}+M_{3} B_{6} e^{-q_{3} y}\right) e^{\imath \omega t} \\
\tilde{t}_{22}(\xi, y, t) & =-\left(N_{1} B_{4} e^{-q_{1} y}+N_{2} B_{5} e^{-q_{2} y}+N_{3} B_{6} e^{-q_{3} y}\right) e^{\imath \omega t}
\end{aligned}
$$

and for $y \leq 0$ as

$$
\begin{aligned}
\tilde{u}_{1}(\xi, y, t) & =\left(a_{1} q_{1} B_{1} e^{q_{1} y}+a_{2} q_{2} B_{2} e^{q_{2} y}+a_{3} q_{3} B_{3} e^{q_{3} y}\right) e^{\imath \omega t}, \\
\tilde{u}_{2}(\xi, y, t) & =\left(b_{1} B_{1} e^{q_{1} y}+b_{2} B_{2} e^{q_{2} y}+b_{3} B_{3} e^{q_{3} y}\right) e^{\imath \omega t}, \\
\tilde{\phi}_{2}(\xi, y, t) & =\left(B_{1} e^{q_{1} y}+B_{2} e^{q_{2} y}+B_{3} e^{q_{3} y}\right) e^{\imath \omega t}, \\
\tilde{m}_{23}(\xi, y, t) & =\left(B_{44} K_{1} / B_{66} A_{11}\right)\left(q_{1} B_{1} e^{q_{1} y}+q_{2} B_{2} e^{q_{2} y}+q_{3} B_{3} e^{q_{3} y}\right) e^{\iota \omega t}, \\
\tilde{t}_{21}(\xi, y, t) & =\left(M_{1} B_{1} e^{q_{1} y}+M_{2} B_{2} e^{q_{2} y}+M_{3} B_{3} e^{q_{3} y}\right) e^{\imath \omega t}, \\
\tilde{t}_{22}(\xi, y, t) & =\left(N_{1} B_{1} e^{q_{1} y}+N_{2} B_{2} e^{q_{2} y}+N_{3} B_{3} e^{q_{3} y}\right) e^{\imath \omega t} .
\end{aligned}
$$

where

$$
\begin{aligned}
M_{i} & =\left(-\iota \xi A_{78} b_{i}+A_{88} a_{i} q_{i}^{2}\right) / A_{11}+K_{1}\left(A_{88}-A_{78}\right) / A_{11}^{2}, \\
N_{i} & =\left(A_{22} b_{i}-\iota \xi A_{12} a_{i}\right) q_{i} / A_{11}
\end{aligned}
$$

and

$$
\begin{aligned}
& B_{1}=B_{4}=F_{o}\left(a_{2}-a_{3}\right) q_{2} q_{3} / \Delta, \\
& B_{2}=B_{5}=F_{o}\left(a_{3}-a_{1}\right) q_{1} q_{3} / \Delta, \\
& B_{3}=B_{6}=F_{o}\left(a_{1}-a_{2}\right) q_{1} q_{2} / \Delta,
\end{aligned}
$$

where

$$
\Delta=2\left[q_{1} q_{2} N_{3}\left(a_{1}-a_{2}\right)+q_{2} q_{3} N_{1}\left(a_{2}-a_{3}\right)+q_{1} q_{3} N_{2}\left(a_{3}-a_{1}\right)\right] .
$$

Thus by (30), the functions $\tilde{u}_{1}, \tilde{u}_{2}, \tilde{\phi}_{3}, \tilde{m}_{23}, \tilde{t}_{21}$ and $\tilde{t}_{22}$ can be determined in the transformed domain and these enable us to find the displacement, microrotation and stresses. 
Particular case: Taking

$$
\begin{array}{ll}
A_{11}=A_{22}=\lambda+2 \mu+K, & A_{77}=A_{88}=\mu+K, \\
A_{12}=\lambda, A_{78}=\mu, & B_{44}=B_{66}=\gamma,
\end{array}
$$

with

$$
-K_{1}=K_{2}=\chi / 2=K,
$$

we obtain the corresponding expressions in the micropolar theory of elasticity.

\section{Inversion of transforms}

The transformed displacements and stresses are functions of $y$, the parameters of Fourier transforms $\xi$, and hence are of the form $\tilde{f}(\xi, y, t)$. To get the function $f(x, y, t)$ in the physical domain, first we invert the Fourier transform using

$$
\begin{aligned}
f(x, y, t) & =\frac{1}{2 \pi} \int_{-\infty}^{\infty} e^{-\iota \xi x} \tilde{f}(\xi, y, t) \mathrm{d} \xi \\
& =\frac{1}{\pi} \int_{0}^{\infty}\left\{\cos (\xi x) f_{e}-\iota \sin (\xi x) f_{o}\right\} \mathrm{d} \xi
\end{aligned}
$$

where $f_{e}$ and $f_{o}$ are even and odd parts of the function $\tilde{f}(\xi, y, t)$ respectively. Thus, (51) gives us function $f(x, y, t)$.

The last step is to evaluate the integral in (51). The method for evaluating this integral by Press et al (1986), which involves the use of Romberg's integration with adaptive step size. This, also uses the results from successive refinement of the extended trapezoidal rule followed by extrapolation of the results to the limit when the step size tends to zero.

\section{Numerical results and discussion}

For numerical computations, we take the following values of relevant parameters for orthotropic micropolar solid:

$$
\begin{aligned}
A_{11} & =13.97 \times 10^{9} \mathrm{~N} / \mathrm{m}^{2}, & & A_{22}=13.75 \times 10^{9} \mathrm{~N} / \mathrm{m}^{2}, \\
A_{77} & =3.0 \times 10^{9} \mathrm{~N} / \mathrm{m}^{2}, & & A_{88}=3.2 \times 10^{9} \mathrm{~N} / \mathrm{m}^{2}, \\
A_{12} & =8.13 \times 10^{9} \mathrm{~N} / \mathrm{m}^{2}, & A_{78} & =2.2 \times 10^{9} \mathrm{~N} / \mathrm{m}^{2}, \\
B_{44} & =0.056 \times 10^{5} \mathrm{~N}, & B_{66} & =0.057 \times 10^{5} \mathrm{~N}, \\
h & =0.01 \times 10^{-2} \mathrm{~m} . & &
\end{aligned}
$$

For comparison with micropolar isotropic solid, following Gauthier (1982), we take the following values of relevant parameters for the case of aluminum epoxy composite as

$$
\begin{aligned}
\rho & =2.19 \times 10^{3} \mathrm{~kg} / \mathrm{m}^{3}, & \lambda & =7.59 \times 10^{9} \mathrm{~N} / \mathrm{m}^{2}, \\
\mu & =1.89 \times 10^{9} \mathrm{~N} / \mathrm{m}^{2}, & K & =0.0149 \times 10^{9} \mathrm{~N} / \mathrm{m}^{2}, \\
\gamma & =0.0268 \times 10^{5} \mathrm{~N}, & j & =0.00196 \times 10^{-4} \mathrm{~m}^{2} .
\end{aligned}
$$




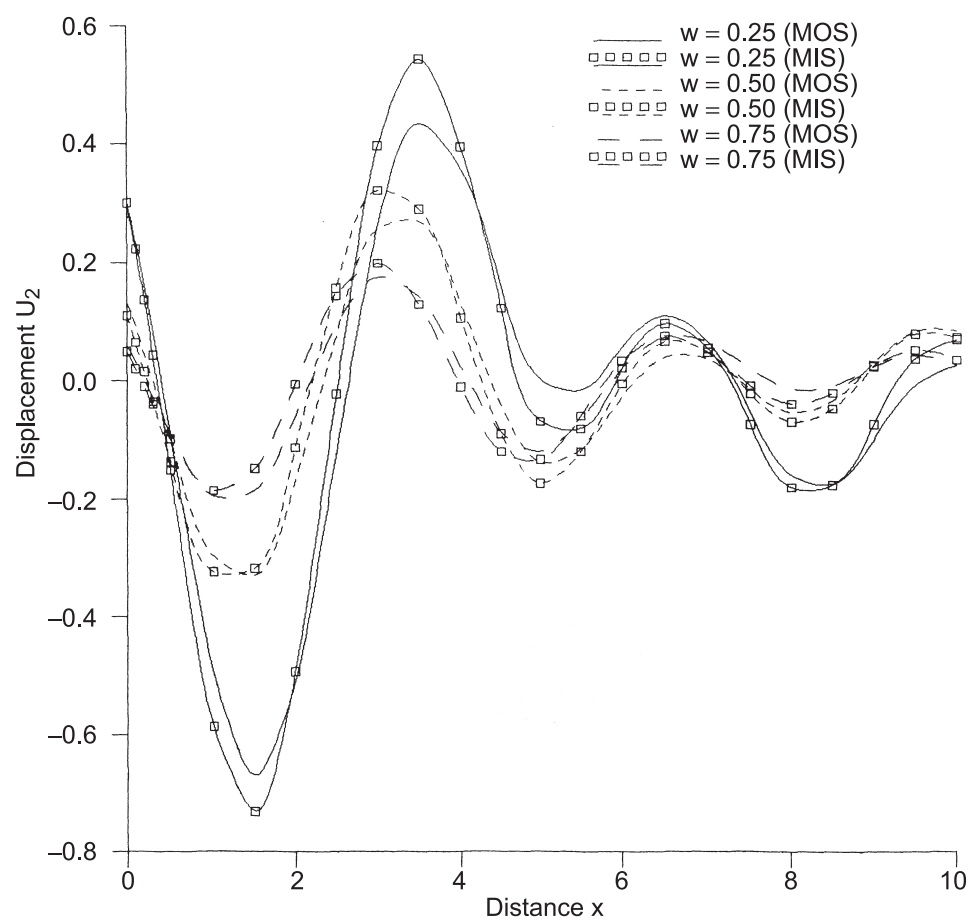

Figure 1. Variation of displacement component $U_{2}\left(=u_{2} / F_{o}\right)$ with distance $x$.

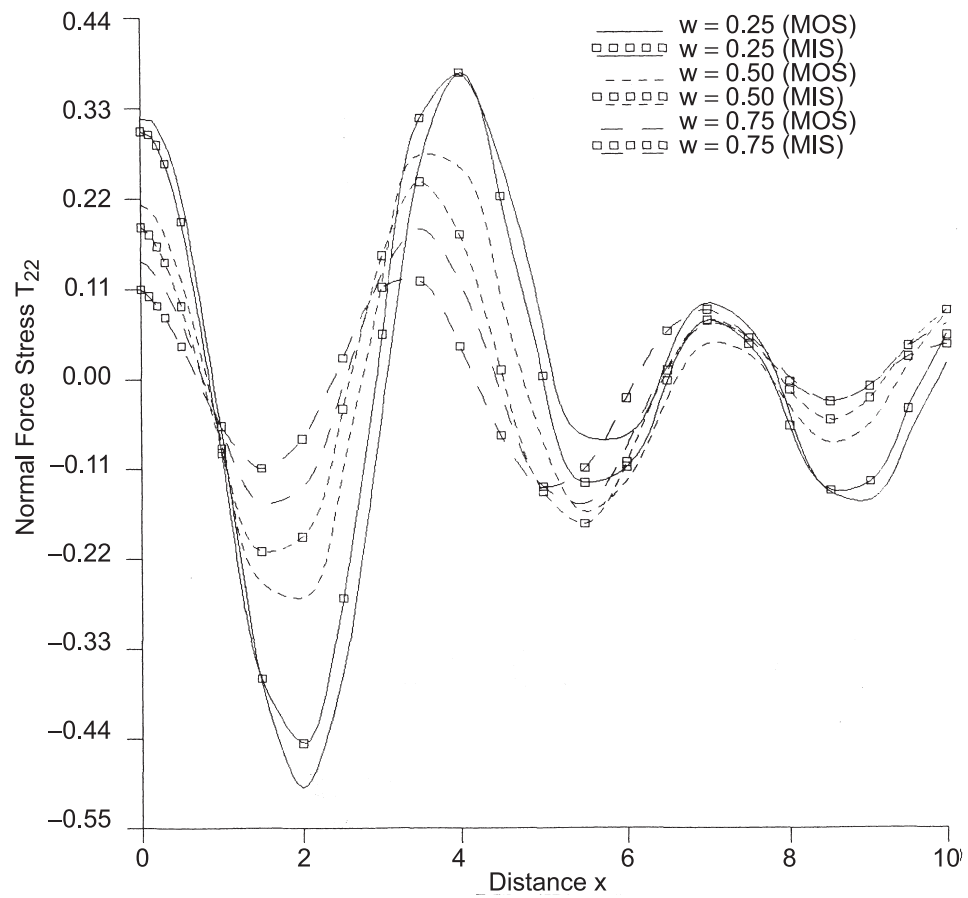

Figure 2. Variation of normal force stress $T_{22}\left(=t_{22} / F_{o}\right)$ with distance $x$. 
The comparison of values of dimensionless displacement component $U_{2}\left[=u_{2} / F_{o}\right]$, normal force stress $T_{22}\left[=t_{22} / F_{o}\right]$ and couple stress $M_{23}\left[=m_{23} / F_{o}\right]$, for micropolar orthotropic solid (MOS) and micropolar isotopic solid (MIS) have been studied. The computations were carried out for three values of dimensionless frequency $\omega=0.25,0.50$ and $\omega=0.75$ for $t=1.0$ at $y=1.0$ in the range $0 \leq x \leq 10$. The solid lines either without centre symbol or with centre symbol represent the variations for $\omega=0.25$ whereas the small dashed lines with or without centre symbol represent the variations for $\omega=0.50$ and large dashed lines with or without centre symbol represent variations for $\omega=0.75$. The curves without centre symbol corresponds to the case of MOS whereas those with centre symbol correspond to the case of MIS.

Figure 1 shows the variations of displacement component $U_{2}$ with $x$. For both cases of MOS and MIS and for all three frequencies, initially the values of $U_{2}$ decrease sharply and as frequency $\omega$ increases from 0.25 to 0.75 , the values of $U_{2}$ increase in the range of $0 \leq x \leq 2.5$ and $6.5 \leq x \leq 10$. The behaviour of variations of $U_{2}$ for both the cases are same.

Figure 2 shows the variations of normal force stress $T_{22}$ with $x$. For all three frequencies, the values of $T_{22}$ for MOS, are greater than those for MIS at the initial value i.e., $x=0$. Initially the values of $T_{22}$ start with sharp decrease then follow oscillatory pattern with reference to $\mathrm{x}$. The minimum stress occur at maximum frequency $\omega=0.75$ in the range $0 \leq x \leq 1$ and $3.5 \leq x \leq 7$ in response of concentrated load.

Figure 3 shows the variations of couple stress $M_{23}$ with $x$. For case MIS , the values of $M_{23}$ have been maximize on multiplying by 10 . Initially, for all three values of $\omega$, the values of $M_{23}$ are less for MIS than those for MOS in the range $0 \leq x \leq 2 \cdot 5$. In both the cases of MOS

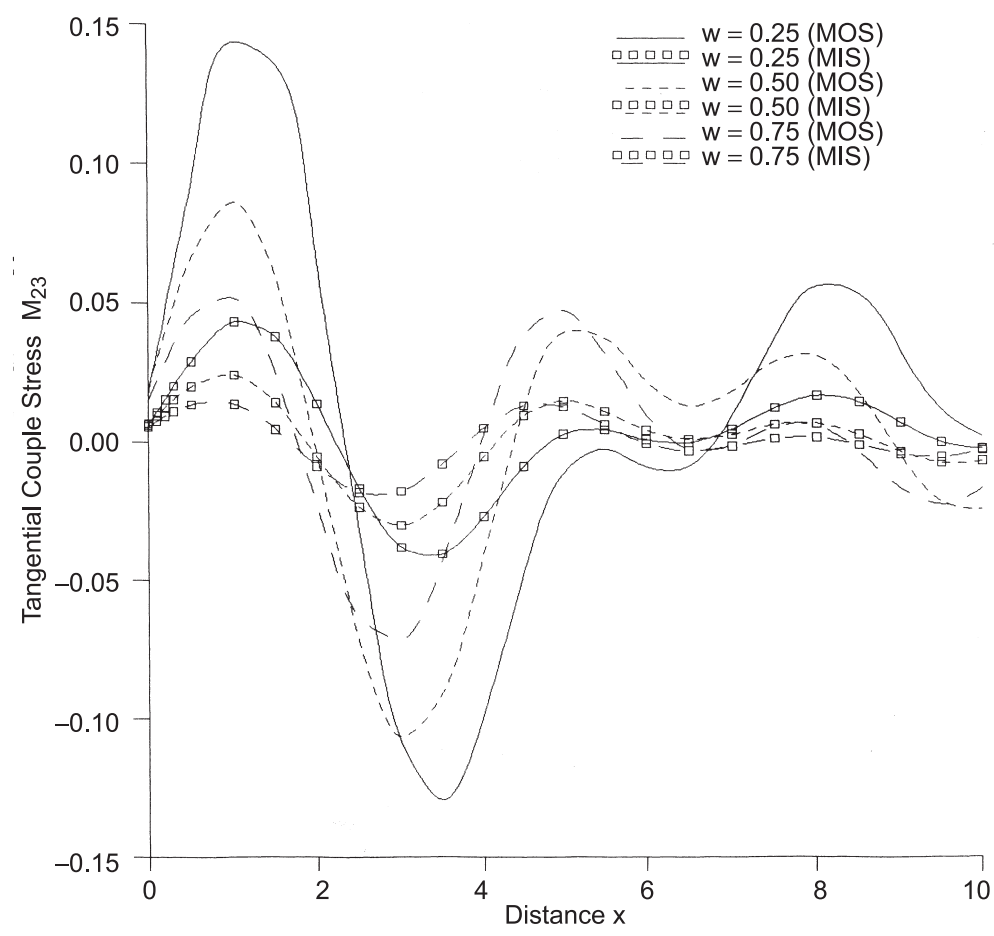

Figure 3. Variation of tangential couple stress $M_{23}\left(=m_{23} / F_{o}\right)$ with distance $x$. 
and MIS, for the maximum value of frequency the value of couple stress is minimum in the ranges $0 \leq x \leq 3$ and $7 \leq x \leq 10$. The behaviour of variations with reference to frequency are oscillating and same for MOS and MIS cases.

\section{Conclusion}

Significant anisotropy effect is obtained on displacement component, force stress and couple stress, for all different frequencies. Near the point of application of load, the frequency plays a major role in all the components.

\section{References}

Cheng Z-Q, He L-H 1995 Micropolar elastic field due to a spherical inclusion. Int. J. Eng. Sci. 33: 389-397

Cheng Z-Q, He L-H 1997 Micropolar elastic field due to a circular inclusion. Int. J. Eng. Sci. 35: 659-668

Erbay H A 2000 An asymptotic theory of thin micropolar plates. Int. J. Eng. Sci. 38: 1497-1516

Eringen A C 1966 linear theory of micropolar elasticity. J. Math. Mech. 15: 909-924

Eringen A C 1968 Theory of micropolar elasticity in fracture (New York, London: Academic Press) 2: $621-729$

Gauthier R D 1982 In Experimental investigations on micropolar media, mechanics of micropolar media (eds) O Brulin, R K T Hsieh. (Singapore: World Scientific)

Iesan D 1973 The plane micropolar strain of orthotropic elastic solids. Arch. Mech. 25: 547-561

Iesan D 1974a Torsion of anisotropic elastic cylinders. ZAMM 54: 773-779

Iesan D 1974b Bending of orthotropic micropolar elastic beams by terminal couples. An. St. Uni. Iasi. 20: $411-418$

Kumar R, Deswal S 2000 Steady-state response of a micropolar generalized thermoelastic half-space to the moving mechanical/thermal loads. Proc. Indian Acad. (Math. Sci.) 119: 449-465

Kumar R, Deswal S 2001 Mechanical and thermal source in a micropolar generalized thermoelastic medium. J. sound and vibrations, 239: 467-488

Kumar R, Singh R, Chadha T K 2001 Eigen value approach to micropolar medium due to impulsive force at the origin. Indian J. Pure Appl. Math. 32: 1127-1144

Mahalabanabis R K, Manna J 1989 Eigenvalue approach to linear micropolar elasticity. Indian J. Pure Appl. Math. 20: 1237-1250

Mahalabanabis R K, Manna J 1997 Eigenvalue approach to the problem of linear micropolar thermoelasticity. J. Indian Acad. Math. 19: 69-86

Nakamura S, Benedict R, Lakes R 1984 Finite elament method for orthotropic micropolar elasticity. Int. J. Eng. Sci. 22: 319-330

Press W H, Teukolsky S A, Vellerlig W T, Flannery B P 1986 Numerical recipes in Fortran 2nd edn (Cambridge: University Press) 